\title{
Locating the ulnar nerve during elbow arthroscopy using palpation is only accurate proximal to the medial epicondyle
}

\author{
Nick F. J. Hilgersom ${ }^{1,2}$ (D) Davide Cucchi ${ }^{3,4}$. Francesco Luceri ${ }^{3,5} \cdot$ Michel P. J. van den Bekerom ${ }^{6} \cdot$ Luke S. Oh $^{2}$. \\ Paolo Arrigoni ${ }^{3,5}$. Denise Eygendaal ${ }^{1,7}$. ESSKA Elbow and Wrist Study Collaborative
}

Received: 11 June 2018 / Accepted: 13 August 2018 / Published online: 23 August 2018

(c) The Author(s) 2018

\begin{abstract}
Purpose Knowledge of ulnar nerve position is of utmost importance to avoid iatrogenic injury in elbow arthroscopy. The aim of this study was to determine how accurate surgeons are in locating the ulnar nerve after fluid extravasation has already occurred, and basing their localization solely on palpation of anatomical landmarks.

Methods Seven cadaveric elbows were used and seven experienced surgeons in elbow arthroscopy participated. An arthroscopic setting was simulated and fluids were pumped into the joint from the posterior compartment for 15 min. For each cadaveric elbow, one surgeon was asked to locate the ulnar nerve solely by palpation of the anatomical landmarks, and subsequently pin the ulnar nerve at two positions: within $5 \mathrm{~cm}$ proximal and another within $5 \mathrm{~cm}$ distal of a line connecting the medial epicondyle and the tip of the olecranon. Subsequently, the elbows were dissected using a standard medial elbow approach and the distances between the pins and ulnar nerve were measured.

Results The median distance between the ulnar nerve and the proximal pins was $0 \mathrm{~mm}$ (range $0-0 \mathrm{~mm}$ ), and between the ulnar nerve and the distal pins was $2 \mathrm{~mm}$ (range 0-10 mm), showing a statistically significant difference $(p=0.009)$. All seven proximally placed pins (100\%) transfixed the ulnar nerve versus two out of seven distally placed pins $(29 \%)(p=0.021)$. Conclusions In a setting simulating an already initiated arthroscopic procedure, the sole palpation of the anatomical landmarks allows experienced elbow surgeons to accurately locate the ulnar nerve only in its course proximal to the medial epicondyle $(7 / 7,100 \%)$, whereas a significantly reduced accuracy is documented when the same surgeons attempt to locate the nerve distal to the medial epicondyle $(2 / 7,29 \% ; p=0.021)$. Current findings support the establishment of a proximal anteromedial portal over a distal anteromedial portal to access the anterior compartment after tissue extravasation has occurred with regard to ulnar nerve safety.
\end{abstract}

Keywords Elbow $\cdot$ Ulnar nerve $\cdot$ Arthroscopy $\cdot$ Physical examination $\cdot$ Nerve injury $\cdot$ Complication $\cdot$ Prevention

The members of ESSKA Elbow and Wrist Study Collaborative are listed in the Acknowledgements section.

Nick F. J. Hilgersom

n.f.hilgersom@amc.uva.nl

1 Department of Orthopaedic Surgery, Academic Medical Center, University of Amsterdam, Amsterdam Movement Sciences, 1105 AZ Amsterdam, The Netherlands

2 Department of Orthopaedic Surgery, Sports Medicine Service, Massachusetts General Hospital, Boston, MA, USA

3 Department of Orthopaedics and Trauma Surgery, University of Bonn, Sigmund-Freud Str. 125, 53127 Bonn, Germany

4 Laboratory of Applied Biomechanics, Department of Biomedical Sciences for Health, Università degli Studi di Milano, Via Mangiagalli 31, 20133 Milan, Italy
5 Clinica Ortopedica CTO, ASST Centro Specialistico Ortopedico Traumatologico Gaetano Pini-CTO, Piazza Cardinal Ferrari 1, 20122 Milan, Italy

6 Department of Orthopaedic Surgery, Onze Lieve Vrouwe Gasthuis, 1091 AC Amsterdam, the Netherlands

7 Department of Orthopaedic Surgery, Amphia Hospital, 4819 EV Breda, the Netherlands 


\section{Abbreviations}

\section{AMP Anteromedial portal}

Distance P Pin-to-nerve distance proximal to the medial epicondyle

Distance D Pin-to-nerve distance distal to the medial epicondyle

SD Standard deviation

\section{Introduction}

Elbow arthroscopy is a safe and established surgical technique, but nerve injuries may occur, also in the hands of experienced surgeons $[2,5,13,15,20]$. In the past two decades, incidences of nerve injury after elbow arthroscopy have been reported between $1.7 \%$ and $5.4 \%$ [7, 13, 15, 18, 20]. Nerves are easily injured during elbow arthroscopy due to their close relation with the elbow joint capsule, which forms only a thin barrier between the arthroscopic working space and the nerves, and arthroscopic portals that pass the nerve with only millimetres of distance $[1,19]$.

Since portal placement is a possible cause of ulnar nerve injury, the knowledge of the distance between the ulnar nerve and the arthroscopy portals is of critical importance for safe surgery: when the nerve follows its common course, the distance to the proximal anteromedial portal (AMP) ranges between 12 and $21 \mathrm{~mm}$, with the smallest reported

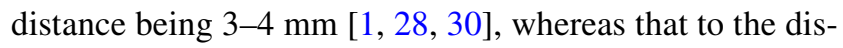
tal AMP ranges between 18 and $25 \mathrm{~mm}$, with the smallest reported distance being $15 \mathrm{~mm}[1,16,30]$.

Therefore, to prevent ulnar nerve injury, the course of the ulnar nerve should always be known prior to AMP placement [11]. Some authors advocate that the ulnar nerve should always be isolated before portal placement, whereas others rely on anatomical landmarks to identify a 'safe working zone' [22, 25].

This "safe working zone" is commonly determined at the beginning of the arthroscopic procedure; however, as the duration of the arthroscopic procedure increases, fluid extravasation and tissue swelling may hide the anatomical landmarks used to locate the nerve or move their projection previously marked on the skin, hence confounding the surgeon and posing additional risk of nerve injury; this may happen, for example, if anterior portals have to be placed after a long procedure in the posterior compartment or if an additional anteromedial portal has to be created to assist an anterior procedure (e.g. arthroscopically assisted radial head or coronoid fracture fixation) several minutes after its beginning.

To the authors' knowledge, the accuracy of locating the ulnar nerve based on palpation has never been quantified before, in particular not after tissue extravasation has occurred. In recent years, Sahajpal et al. [25] and Park et al.
[22] proposed the use of an algorithm based on palpation of the ulnar nerve to decide what portal placement technique to use to avoid ulnar nerve injury and successfully used this algorithm in clinical practice; however, this algorithm is applied prior to tissue extravasation.

The aim of this study was to determine how accurate surgeons are in locating the ulnar nerve after tissue extravasation has already occurred, moreover, basing their localization solely on palpation of anatomical landmarks. The study hypothesis is that the ulnar nerve can be located precisely by more than $75 \%$ of the surgeons based on palpation of anatomical landmarks.

\section{Materials and methods}

Seven fresh-frozen upper limb cadaveric specimens were prepared to mimic an arthroscopic setting. Care was taken in evaluating the specimens for any visible signs of previous trauma, gross instability, deformity or signs of previous surgery. None of the elbow specimens had a (sub)luxating or transposed ulnar nerve. Prior to the beginning of the study, three lines were marked on the skin: the first line connecting the olecranon's tip with the medial epicondyle; the second and the third parallel to the first one, $5 \mathrm{~cm}$ proximal and $5 \mathrm{~cm}$ distal. These lines were used to determine the regions in which the ulnar nerve would have to be located (Fig. 1).

Arthroscopy was performed with the elbow positioned at $90^{\circ}$ of flexion, with the hand and forearm hanging free with only gravity force. Standard posterolateral and midlateral

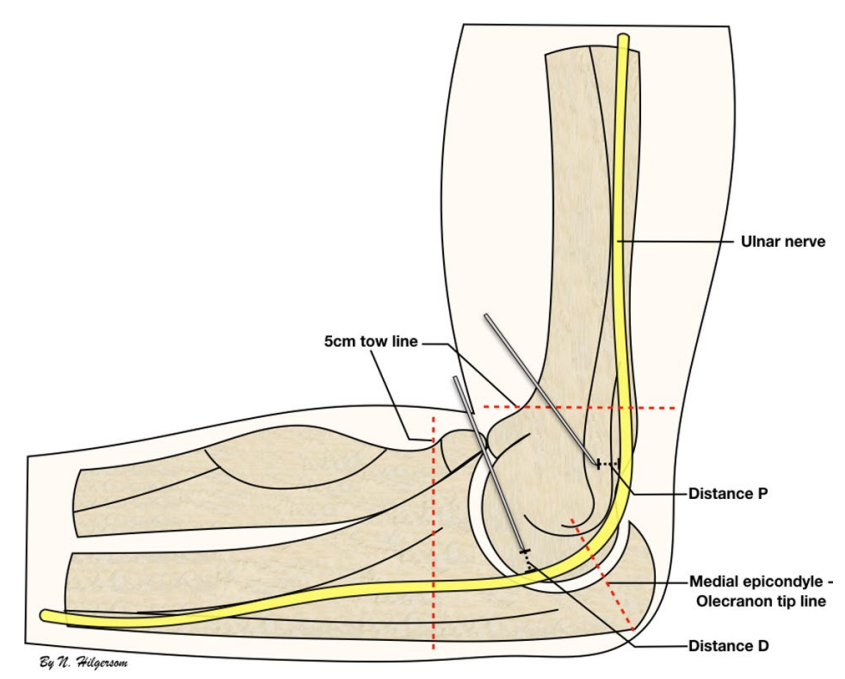

Fig. 1 Schematic representation of the setup and pinning of the ulnar nerve. Schematic representation of the medial side of the elbow showing the line between the medial epicondyle and the olecranon tip, the tow lines, distance $\mathrm{P}$ [distance between the proximal pin and ulnar nerve, $(\mathrm{mm})$ ] and distance D [distance between the distal pin and ulnar nerve $(\mathrm{mm})]$ 
portals were established. To reproduce a realistic arthroscopic setting with tissue swelling, fluids were pumped in the joint for $15 \mathrm{~min}$, without moving the instruments to the anterior compartment and without creating anterior portals. Subsequently, seven fellowship-trained elbow surgeons were asked to locate the ulnar nerve only by palpating anatomical landmarks (e.g. medial epicondyle, medial intermuscular septum). For each cadaveric specimen, one surgeon was asked to try to transfix the ulnar nerve with two pins, in two different locations: first within $5 \mathrm{~cm}$ proximal to the medial epicondyle, and then within $5 \mathrm{~cm}$ distal to it, as previously marked with parallel lines on the skin. After each specimen was transfixed with two pins, all specimens were dissected using a standard medial approach to expose the cubital tunnel. The ulnar nerve was then identified without removal of the pins and two distances were measured with a graduated calliper allowing accurate measurements with $1 \mathrm{~mm}$ accuracy: between the proximal pin and the ulnar nerve (distance $\mathrm{P}$ ), and between the distal pin and the ulnar nerve (distance D). An external observer, not included among the examiners and not involved in the statistical evaluation of the collected data, measured each distance in triplicate. The averages of all three measurements per distance were used for statistical analysis. In addition, the trans-epicondylar distance was measured in each cadaver specimen to provide an indication about the elbow size.

Institutional approval of the study protocol was obtained by the Nicola's Foundation \& ICLO Research Center (ID10607).

\section{Statistical analysis}

Data analysis was performed using Stata, version 14.0 (StataCorp LP, College Station, TX, USA). The pin-to-nerve distances are reported as mean and standard deviation (SD) or median and range, depending on the normality of the data. In bivariate analysis, a Student's $t$ test or Mann-Whitney $U$ test, depending on normality of the data, was used to compare distance $\mathrm{P}$ and distance $\mathrm{D}$. In addition, the pin-to-nerve distances were dichotomized into hits (distance of $0 \mathrm{~mm}$ ) and misses (distance $>0 \mathrm{~mm}$ ). A Fisher's exact test was used to compare the proportions of hits proximal and distal to the line between the medial epicondyle and olecranon tip. A $p$ value of $<0.05$ was considered statistically significant.

Since the number of anatomical studies dealing with the mutual relation between anteromedial portals and ulnar nerve is limited, the sample size for this study was based on previous publications regarding anatomical dissections of the more frequently studied posterior interosseous nerve: this was successfully investigated also on relatively small groups of specimens in open, arthroscopic and imagingcontrolled studies [3, 9, 17, 29]. Based on these experiences, a minimum of five specimens was considered as suitable to conduct this study. The number of experienced elbow arthroscopy surgeons available at the moment of study conduction limited the sample size to a final number of seven.

\section{Results}

Seven fresh-frozen elbow specimens (females $86 \%$, left elbow $43 \%$, median inter-epicondylar distance: $6 \mathrm{~mm}$ ) were evaluated by seven surgeons. The demographics of the surgeons are shown in Table 1.

All seven pins (100\%) placed proximally to the medial epicondyle transfixed the ulnar nerve (distance $\mathrm{P}=0 \mathrm{~mm}$ ). Only two out of seven (29\%) pins placed distally to the medial epicondyle transfixed the ulnar nerve. The median distance $\mathrm{D}$ was $2 \mathrm{~mm}$ (range $0-10 \mathrm{~mm}$ ). Bivariate analysis showed a statistically significant difference between both distance $\mathrm{P}$ and distance $\mathrm{D}(p=0.0091)$, and the number of pins transfixing the ulnar nerve proximal and distal to the line between the medial epicondyle and olecranon top $(p=0.021)$ (Table 2).

\section{Discussion}

The main finding of this study is that in a setting simulating an already initiated arthroscopic procedure, the sole palpation of the anatomical landmarks allows experienced elbow surgeons to accurately locate the ulnar nerve only in its course proximal to the medial epicondyle (7/7, 100\%), whereas a significantly reduced accuracy in the localization of the nerve is documented when the same surgeons attempt to locate the nerve distal to the medial epicondyle $(2 / 7,29 \%$; $p=0.021)$. Therefore, our hypothesis that more than $75 \%$ of surgeons can accurately locate the ulnar nerve by palpation of anatomical landmarks after fluid extravasation is

Table 1 Demographics of the surgeons

\begin{tabular}{lllll}
\hline Expert & Gender & Age (years) & $\begin{array}{l}\text { Experi- } \\
\text { ence } \\
\text { (years) }\end{array}$ & $\begin{array}{l}\text { Number of } \\
\text { arthroscopies (past } \\
12 \text { months) }\end{array}$ \\
\hline 1 & Male & 41 & 10 & 80 \\
2 & Male & 48 & 14 & 24 \\
3 & Female & 48 & 17 & 40 \\
4 & Male & 47 & 15 & 100 \\
5 & Male & 45 & 9 & 50 \\
6 & Male & 61 & 27 & 70 \\
7 & Male & 39 & 5 & 45 \\
& Median & 47 & 14 & 50 \\
& Range & $39-61$ & $5-27$ & $24-100$ \\
\hline
\end{tabular}


Table 2 The pin-to-nerve distances measured for each surgeon

\begin{tabular}{|c|c|c|c|c|c|}
\hline \multicolumn{3}{|l|}{ Surgeon data } & \multicolumn{3}{|c|}{ Elbow specimen data $(n=8)$} \\
\hline Surgeon & Distance P $(\mathrm{mm})$ & $\begin{array}{l}\text { Distance D } \\
(\mathrm{mm})\end{array}$ & Laterality & Sex & $\begin{array}{l}\text { Trans-epicon- } \\
\text { dylar distance } \\
(\mathrm{cm})\end{array}$ \\
\hline 1 & 0 & 0 & Right & Male & 6 \\
\hline 2 & 0 & 10 & Left & Female & 5.7 \\
\hline 3 & 0 & 0 & Right & Female & 6.6 \\
\hline 4 & 0 & 2 & Left & Female & 8.2 \\
\hline 5 & 0 & 1 & Right & Female & 5.8 \\
\hline 6 & 0 & 5 & Left & Female & 4.9 \\
\hline 7 & 0 & 2 & Right & Female & 6.4 \\
\hline Median & 0 & 2 & & & \\
\hline Range & $0-0$ & $0-10$ & & & \\
\hline $\begin{array}{l}\text { Mann-Whitney } \\
U \text { test }\end{array}$ & $p=0.0091 *$ & & & & \\
\hline
\end{tabular}

*Distance $\mathrm{P}=$ pin-to-nerve distance proximal to the medial epicondyle

*Distance $\mathrm{D}=$ pin-to-nerve distance distal to the medial epicondyle confirmed for the proximal localization and is rejected for the distal one.

Nerves at risk during elbow arthroscopy are the radial, median, ulnar, lateral antebrachial cutaneous and medial antebrachial cutaneous nerve. Approximately, 38-42\% of nerve injuries associated with elbow arthroscopy involve the ulnar nerve $[5,15]$. The ulnar nerve is frequently injured by direct trauma, which can occur by posteromedial or AMP placement (Fig. 2), or using motorized instruments inside the joint in close proximity to the medial capsule $[6-8,10$, 12]. The latter may lead to accidental entanglement or suction injury of the ulnar nerve, as the medial capsule forms only a thin barrier between the arthroscopic working space and the nerve. The posteromedial portal is generally not used, as it directly overlies the ulnar nerve [21,28].

Anatomical studies describe that the ulnar nerve, after traversing through the arcade of Struthers, runs posteriorly to the medial intermuscular septum in the upper arm and then courses posteriorly to the medial epicondyle to enter the cubital tunnel (Fig. 3) [14, 24, 27]. However, in the presence of a luxating or subluxating ulnar nerve, a previously transposed ulnar nerve or in the rare cases in which the ulnar nerve runs anterior to the medial epicondyle, the ulnar nerve may not be where expected and the nerve-to-portal distances may be smaller $[8,26]$. These instances pose extra risk for iatrogenic ulnar nerve injury during anteromedial portal placement (Figs. 2, 3).

Based on the high accuracy found for proximal localization of the ulnar nerve, experienced elbow surgeons appeared capable of determining a safe proximal AMP site using palpation of the ulnar nerve only, also after beginning of the arthroscopic procedure and joint irrigation. The findings of our study support the algorithm proposed by Park

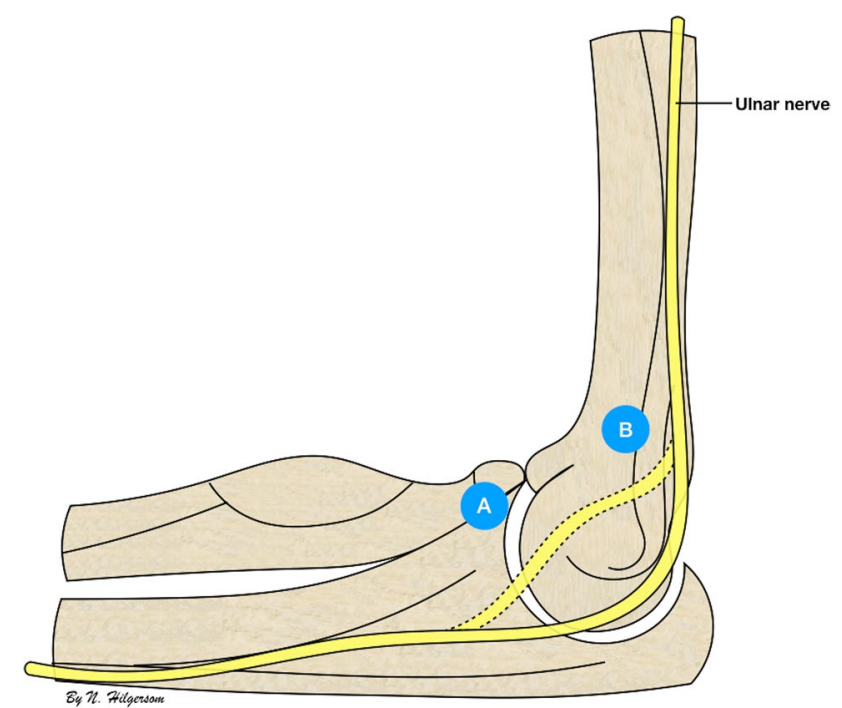

Fig. 2 The ulnar nerve and anteromedial portals. Schematic representation of the medial side of the elbow showing the anatomic course of the ulnar nerve (continuous line) and a luxating or transposed ulnar nerve (dashed line) in relation to the distal anteromedial portal (A), $2 \mathrm{~cm}$ distal and $2 \mathrm{~cm}$ anterior to the medial epicondyle, and proximal anteromedial portal (B), $2 \mathrm{~cm}$ proximal and just anterior to the medial intermuscular septum

et al. [22], originally designed to guide safe proximal AMP placement in elbows with a transposed ulnar nerve. In this algorithm, patients are subdivided based on the certainty with which the ulnar nerve can be located (certain versus uncertain). In case the ulnar nerve is palpable with certainty, the portal is placed in a standard antegrade fashion, approximately $1 \mathrm{~cm}$ away from the ulnar nerve, while holding the 


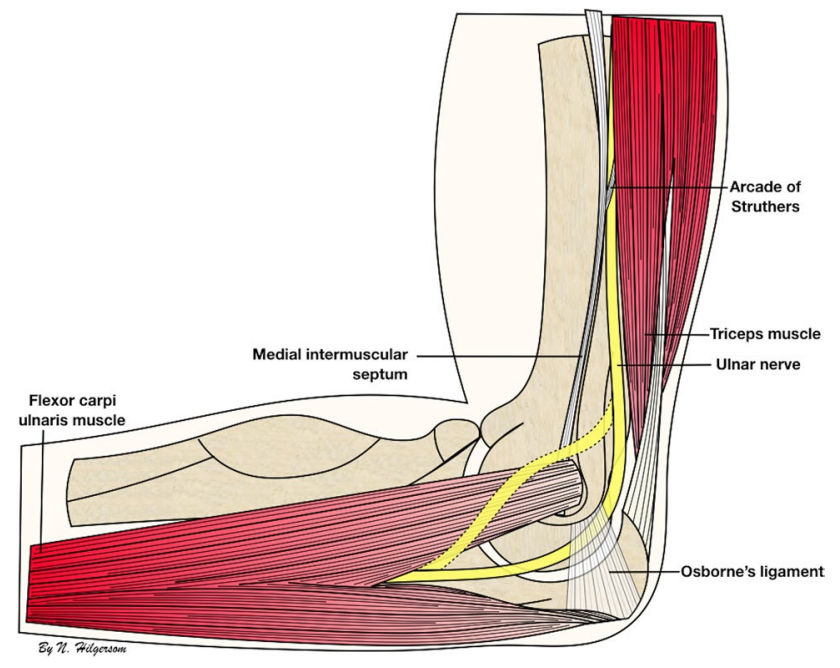

Fig. 3 Ulnar nerve anatomy. Schematic representation of the medial side of the elbow showing the anatomic course of the ulnar nerve (continuous line) and the course of a luxating or transposed ulnar nerve (dashed line), including relevant anatomic features along its course

ulnar nerve posteriorly or anteriorly if mobile. However, in case the location of the ulnar nerve is doubtful, following this algorithm, a 1-3 cm skin incision with blunt dissection onto the capsule is recommended before portal placement, to prevent iatrogenic injury to the ulnar nerve. Using this algorithm, under the assumption that all surgeons in our study were certain about the proximal location of the ulnar nerve, none of the surgeons would have injured the ulnar nerve while placing a proximal AMP after palpation of the ulnar nerve.

Distal localization of the ulnar nerve was significantly less accurate among the experienced elbow surgeons in this study, with a median pin-to-nerve distance of $2 \mathrm{~mm}$ (range $0-10 \mathrm{~mm}$ ). This finding supports current clinical practice, in which, ever since the introduction of the proximal AMP by Poehling et al. [23], the distal anteromedial portal was abandoned as a starting portal. The distal AMP is currently only used as a secondary portal, established using an insideout technique to avoid injury to the median nerve. The significant lower accuracy in locating the ulnar nerve in this study further supports the use of an inside-out technique for establishment of a distal AMP, to protect the median as well as the ulnar never from iatrogenic damage.

The fact that the measurements in this study were performed after $15 \mathrm{~min}$ of joint irrigation further supports this indication, since fluid extravasation and soft tissue swelling appeared to critically compromise the palpability of the ulnar nerve in its distal portion, leading the majority of the involved surgeons to miss the ulnar nerve. Therefore, if the creation of an accessory anteromedial portal is necessary after beginning of the arthroscopic procedure, it is recommended to isolate and identify the ulnar nerve.

The difference in accuracy of locating the ulnar nerve with palpation proximally and distally may be explained by numerous factors: the anatomic trajectory of the ulnar nerve, the presence of the medial intermuscular septum and the tissue swelling. The ulnar nerve runs relatively superficial proximal to the medial epicondyle, as it is not covered by any fascia or ligament after it has passed Struther's ligament, while distal to the medial epicondyle, the ulnar nerve traverses the cubital tunnel underneath Osborne's ligament and Osborne's fascia, and continues beneath the aponeurosis of the humeral and ulnar heads of the flexor carpi ulnaris muscle [14, 24, 27], possibly making it harder to palpate. In addition, the well-palpable medial intermuscular septum can be used as a reliable anatomic landmark proximal of the medial epicondyle, whereas a similar anatomic reference distal to the medial epicondyle is lacking. Finally, in a setting simulating a lateral decubitus position with the elbow positioned at $90^{\circ}$ of flexion and the forearm hanging free with only gravity force, the extravasation of fluid into the periarticular soft tissues may be more prominent in the distal part.

Several limitations should be considered for this study. First, we examined a relatively small group of experienced elbow surgeons. Therefore, the accuracy of ulnar nerve identification found in this study may not apply to less experienced surgeons. Claessen et al. [4] found a significantly higher complication rate with portal placement by novice surgeons compared to reported complication rates by experienced elbow surgeons $[7,18]$. Secondly, the number of surgeons participating in this study and the number of cadaveric specimens were small.

To the best of our knowledge, this is the first study examining the accuracy of surgeons in ulnar nerve localization by palpation in a simulated arthroscopic setting after several minutes of joint irrigation. Our findings confirm that experienced elbow surgeons are capable of accurate localization of the ulnar nerve proximal to the medial epicondyle using solely palpation after tissue extravasation has occurred, allowing safe proximal AMP placement based solely on palpation of the ulnar nerve in the swollen elbow. Their localization of the ulnar nerve distal to the medial epicondyle was significantly less accurate, supporting the current practice of using an inside-out technique for distal AMP placement. The relevance of the current study for daily clinical practise is that it shows the preferential use of a proximal anteromedial portal over a distal anteromedial portal to access the anterior compartment after tissue extravasation had occurred with regard to ulnar nerve safety. Nonetheless, it remains paramount for a surgeon to remain self-critical, and if doubtful about the location of the ulnar nerve to use a skin incision and blunt 
dissection onto the capsule, to avoid the risk of iatrogenic nerve injury because of portal placement.

\section{Conclusions}

In a setting simulating an already initiated arthroscopic procedure, the sole palpation of the anatomical landmarks allows experienced elbow surgeons to accurately locate the ulnar nerve only in its course proximal to the medial epicondyle $(7 / 7,100 \%)$, whereas a significantly reduced accuracy in the localization of the nerve is documented when the same surgeons attempt to locate the nerve distal to the medial epicondyle $(2 / 7,29 \% ; p=0.021)$. Relevance for daily clinical practice is the support for use of a proximal anteromedial portal over a distal anteromedial portal to gain access to the anterior elbow compartment after tissue extravasation has occurred with regard to ulnar nerve safety.

Acknowledgements We would like to offer our special gratitude to all surgeons of the ESSKA Elbow and Wrist Study Collaborative who participated in this study.

ESSKA Elbow and Wrist Study Collaborative: Raul Barco (Spain), Andrea Celli (Italy), Enrico Guerra (Italy), Nicolas Holzer (Switzerland), Andreas Lenich (Germany), Simone Nicoletti (Italy), Luigi Pederzini (Italy), Hakan Turan Çift (Turkey), Kilian Wegmann (Germany), Oskar Zupanc (Slovenia), Denise Eygendaal (Netherlands), Paolo Arrigoni (Italy).

Permissions All images in the article were created by Nick F. J. Hilgersom. They are licensed under Creative Commons Attribution NonCommercial 4.0 (https://creativecommons.org/licenses/by-nc/4.0/deed. $\mathrm{nl})$. This means that everyone is free to share and adapt the images as long as they attribute Nick F. J. Hilgersom as the author and do so for non-commercial purposes. You can contact Nick F. J. Hilgersom for additional permissions.

Author contributions NFJH is responsible for study design, data analysis, data interpretation, manuscript preparation, creation of figures and editing. DC and FL are responsible for study design, data collection, data interpretation and providing feedback on the manuscript. MPJB, LSO, PA and DE were responsible for study design, data interpretation, providing feedback on the manuscript and clinical insight.

Funding This research received no specific grant from any funding agency in the public, commercial or not-for-profit sectors.

\section{Compliance with ethical standards}

Conflict of interest The authors, their immediate relatives, and any research foundations with which they are affiliated have not received any financial payments or other benefits from any commercial entity related to the subject of this article.

Ethical approval Nicola's Foundation and ICLO Research Center (ID10607).

Informed consent Not required (cadaver study).
Open Access This article is distributed under the terms of the Creative Commons Attribution 4.0 International License (http://creativeco mmons.org/licenses/by/4.0/), which permits unrestricted use, distribution, and reproduction in any medium, provided you give appropriate credit to the original author(s) and the source, provide a link to the Creative Commons license, and indicate if changes were made.

\section{References}

1. Adolfsson L (1994) Arthroscopy of the elbow joint: a cadaveric study of portal placement. J Shoulder Elbow Surg 3:53-61

2. Andrews JR, Carson WG (1985) Arthroscopy of the elbow. Arthroscopy 1:97-107

3. Arrigoni P, Cucchi D, Guerra E, Marinelli A, Menon A, Randelli PS, Pederzini LA, Committee SUL (2017) Distance of the posterior interosseous nerve from the radial head during elbow arthroscopy: an anatomical study. Joints 5:147-151

4. Claessen F, Kachooei AR, Kolovich GP, Buijze GA, Oh LS, van den Bekerom MPJ, Doornberg JN (2017) Portal placement in elbow arthroscopy by novice surgeons: cadaver study. Knee Surg Sports Traumatol Arthrosc 25:2247-2254

5. Desai MJ, Mithani SK, Lodha SJ, Richard MJ, Leversedge FJ, Ruch DS (2016) Major peripheral nerve injuries after elbow arthroscopy. Arthroscopy 32:999-1002 e1008

6. Dumonski ML, Arciero RA, Mazzocca AD (2006) Ulnar nerve palsy after elbow arthroscopy. Arthroscopy 22:577 e571-e573

7. Elfeddali R, Schreuder MH, Eygendaal D (2013) Arthroscopic elbow surgery, is it safe? J Shoulder Elbow Surg 22:647-652

8. Gay DM, Raphael BS, Weiland AJ (2010) Revision arthroscopic contracture release in the elbow resulting in an ulnar nerve transection: a case report. J Bone Jt Surg Am 92:1246-1249

9. Hackl M, Wegmann K, Lappen S, Helf C, Burkhart KJ, Muller LP (2015) The course of the posterior interosseous nerve in relation to the proximal radius: is there a reliable landmark? Injury 46:687-692

10. Hahn M, Grossman JA (1998) Ulnar nerve laceration as a result of elbow arthroscopy. J Hand Surg Br 23:109

11. Hilgersom NF, Oh LS, Flipsen M, Eygendaal D, van den Bekerom MP (2017) Tips to avoid nerve injury in elbow arthroscopy. World J Orthop 8:99-106

12. Hilgersom NFJ, van Deurzen DFP, Gerritsma CLE, van der Heide HJL, Malessy MJA, Eygendaal D, van den Bekerom MPJ (2018) Nerve injuries do occur in elbow arthroscopy. Knee Surg Sports Traumatol Arthrosc 26:318-324

13. Jinnah AH, Luo TD, Wiesler ER, Li Z, Poehling GG, Tuohy CJ, Graves BR, Freehill MT, Papadonikolakis A (2018) Peripheral nerve injury after elbow arthroscopy: an analysis of risk factors. Arthroscopy 34:1447-1452

14. Kane E, Kaplan EB, Spinner M (1973) Observations of the course of the ulnar nerve in the arm. Ann Chir 27:487-496

15. Kelly EW, Morrey BF, O'Driscoll SW (2001) Complications of elbow arthroscopy. J Bone Jt Surg Am 83-A:25-34

16. Lindenfeld TN (1990) Medial approach in elbow arthroscopy. Am J Sports Med 18:413-417

17. Links AC, Graunke KS, Wahl C, Green JR 3rd, Matsen FA 3rd (2009) Pronation can increase the pressure on the posterior interosseous nerve under the arcade of Frohse: a possible mechanism of palsy after two-incision repair for distal biceps rupture-clinical experience and a cadaveric investigation. J Shoulder Elbow Surg 18:64-68 
18. Marti D, Spross C, Jost B (2013) The first 100 elbow arthroscopies of one surgeon: analysis of complications. J Shoulder Elbow Surg 22:567-573

19. Miller CD, Jobe CM, Wright MH (1995) Neuroanatomy in elbow arthroscopy. J Shoulder Elbow Surg 4:168-174

20. Nelson GN, Wu T, Galatz LM, Yamaguchi K, Keener JD (2014) Elbow arthroscopy: early complications and associated risk factors. J Shoulder Elbow Surg 23:273-278

21. Ohuchi H, Torres RJL, Shinga K, Ichikawa K, Kato Y, Hattori S, Yamada S (2017) Ultrasound-assisted posteromedial portal placement of the elbow joint to prevent ulnar nerve injury. Arthrosc Tech 6:e1087-e1091

22. Park SE, Bachman DR, O'Driscoll SW (2016) The safety of using proximal anteromedial portals in elbow arthroscopy with prior ulnar nerve transposition. Arthroscopy 32:1003-1009

23. Poehling GG, Whipple TL, Sisco L, Goldman B (1989) Elbow arthroscopy: a new technique. Arthroscopy 5:222-224

24. Polatsch DB, Melone CP Jr, Beldner S, Incorvaia A (2007) Ulnar nerve anatomy. Hand Clin 23:283-289
25. Sahajpal DT, Blonna D, O’Driscoll SW (2010) Anteromedial elbow arthroscopy portals in patients with prior ulnar nerve transposition or subluxation. Arthroscopy 26:1045-1052

26. Satteson ES, Li Z (2015) Anteriorly positioned ulnar nerve at the elbow: a rare anatomical event: case report. J Hand Surg Am 40:984-986

27. Spinner M, Spencer PS (1974) Nerve compression lesions of the upper extremity. A clinical and experimental review. Clin Orthop Relat Res 104:46-67

28. Stothers K, Day B, Regan WR (1995) Arthroscopy of the elbow: anatomy, portal sites, and a description of the proximal lateral portal. Arthroscopy 11:449-457

29. Strachan JC, Ellis BW (1971) Vulnerability of the posterior interosseous nerve during radial head resection. J Bone Jt Surg Br 53:320-323

30. Unlu MC, Kesmezacar H, Akgun I, Ogut T, Uzun I (2006) Anatomic relationship between elbow arthroscopy portals and neurovascular structures in different elbow and forearm positions. J Shoulder Elbow Surg 15:457-462 